PREPARED FOR THE U.S. DEPARTMENT OF ENERGY, UNDER CONTRACT DE-AC02-76CH03073

PPPL-3839

PPPL-3839

UC-70

Finite-duration Seeding Effects in Powerful Backward Raman Amplifiers

by

N.A. Yampolsky, V.M. Malkin, and N.J. Fisch

July 2003

$\left.\stackrel{M}{M}\right|_{\substack{\text { PRInCETON PLASIMA } \\ \text { PHYSICS LABORATORY }}} ^{D}$

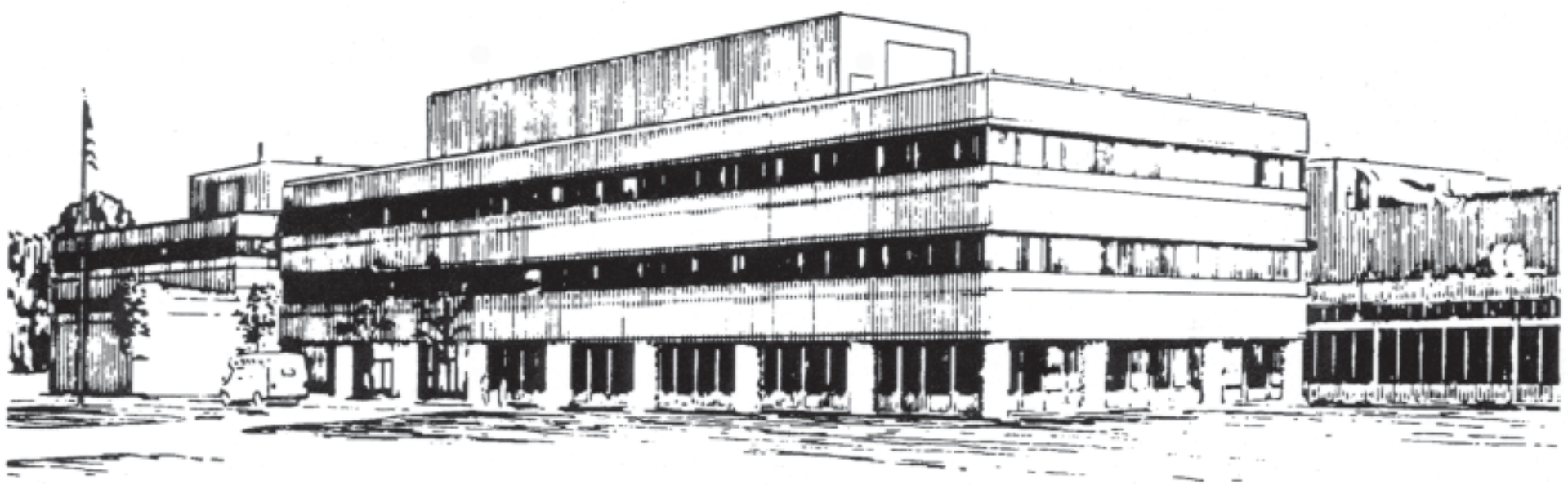

PRINCETON PLASMA PHYSICS LABORATORY PRINCETON UNIVERSITY, PRINCETON, NEW JERSEY 


\section{PPPL Reports Disclaimer}

This report was prepared as an account of work sponsored by an agency of the United States Government. Neither the United States Government nor any agency thereof, nor any of their employees, makes any warranty, express or implied, or assumes any legal liability or responsibility for the accuracy, completeness, or usefulness of any information, apparatus, product, or process disclosed, or represents that its use would not infringe privately owned rights. Reference herein to any specific commercial product, process, or service by trade name, trademark, manufacturer, or otherwise, does not necessarily constitute or imply its endorsement, recommendation, or favoring by the United States Government or any agency thereof. The views and opinions of authors expressed herein do not necessarily state or reflect those of the United States Government or any agency thereof.

\section{Availability}

This report is posted on the U.S. Department of Energy's Princeton Plasma Physics Laboratory Publications and Reports web site in Fiscal Year 2003. The home page for PPPL Reports and Publications is: http://www.pppl.gov/pub_report/

DOE and DOE Contractors can obtain copies of this report from:

U.S. Department of Energy

Office of Scientific and Technical Information

DOE Technical Information Services (DTIS)

P.O. Box 62

Oak Ridge, TN 37831

Telephone: (865) 576-8401

Fax: (865) 576-5728

Email: reports@adonis.osti.gov

This report is available to the general public from:

National Technical Information Service

U.S. Department of Commerce

5285 Port Royal Road

Springfield, VA 22161

Telephone: $1-800-553-6847$ or

(703) $605-6000$

Fax: (703) 321-8547

Internet: http://www.ntis.gov/ordering.htm 


\title{
Finite-duration seeding effects in powerful backward Raman amplifiers
}

\author{
N. A. Yampolsky, V. M. Malkin, and N. J. Fisch \\ Department of Astrophysical Sciences, Princeton University, Princeton, NJ 08544
}

(July 3, 2003)

\begin{abstract}
In the process of backward Raman amplification (BRA), the leading layers of the seed laser pulse can shadow the rear layers, thus weakening the effective seeding power and affecting parameters of output pulses in BRA. We study this effect numerically and also analytically by approximating the pumped pulse by the " $\pi$-pulse" manifold of self-similar solutions. We determine how the pumped pulse projection moves within the $\pi$-pulse manifold, and describe quantitatively the effective seeding power evolution. Our results extend the quantitative theory of BRA to regimes where the effective seeding power varies substantially during the amplification. These results might be of broader interest, since the basic equations are general equations for resonant 3-wave interactions.
\end{abstract}

PACS numbers: 52.38.Bv, 52.35.Mw, 42.65.Yj, 42.65.Dr

\section{INTRODUCTION}

Backward Raman amplification might be utilized to achieve ultrahigh laser powers (Ref. [1]). In this scheme, a short pumped pulse consumes essentially all of a long incident pump power through the resonant stimulated backward Raman scattering in plasma and contracts as its amplitude grows. The amplification/compression is fast enough to reach nearly relativistic pumped pulse intensities, like $10^{17} \mathrm{~W} / \mathrm{cm}^{2}$ for $\lambda=1 \mu \mathrm{m}$-wavelength radiation, within times shorter than it takes for filamentation instabilities to develop. Such a nonfocused intensity would be $10^{5}$ times higher than currently available through the chirp pulse amplification technique (see, for instance, Ref. [2]). For shorter wavelength lasers, this factor would be even higher (see Refs. [1,3]). Moreover, backward Raman amplifier output pulses can maintain high focusability of relatively low intensity input seeds (Refs. [1,4]), which makes feasible the same huge increase in achievable focused intensities as well, say up to $10^{25} \mathrm{~W} / \mathrm{cm}^{2}$ for $\lambda=1 \mu \mathrm{m}$-wavelength output pulse focused from $10 \mathrm{~cm}$ to $10 \mu \mathrm{m}$ diameter.

The amplification mechanism can be briefly outlined as follows: Upon the short seed pulse arrival to any given layer of plasma, beatings of the pump and seed pulses resonantly generate there a fresh Langmuir wave. The short seed quickly leaves this place and proceeds further being nearly not affected by the interaction, while the Langmuir wave stays at the place, backscattering the pump and growing. The backscattered light, called further "the pumped pulse" has the same frequency as the seed pulse and propagates in the same direction trailing the seed and growing until the pump is depleted.

In a broad parameter range of interest, BRA along each ray is an essentially one-dimensional process. As long as effects of the input seed pulse finite duration can be neglected, the pumped pulse evolution approaches, at an advance nonlinear stage, a self-similar regime known as " $\pi$-pulse" regime (see Refs. [5,3]). The $\pi$-pulse amplitude grows linearly with the amplification distance while the $\pi$-pulse duration decreases inversely with the amplitude. There is a family of such self-similar solutions that can be conveniently characterized by location $z_{s}$ of the (physically zero-duration) seed and location $z_{M}$ of the pumped pulse intensity maximum. This maximum propagates behind the original seed, approaching it as the pumped pulse grows and contracts. When the pumped pulse duration becomes comparable with the original seed duration, the amplification occurring from the front and rear layers of the original seed become noticeably different at the location of pumped pulse maximum. The contribution of rear layers of the original seed are then noticeably shadowed by that of the leading layers, which quickly deplete the pump. This reduces the area of the original seed, which effectively contributes to the amplification. We will define further an effective dimensionless area under the seed pulse envelope $\epsilon_{\text {eff }}$, which can be called an "effective seeding power", and express it in the terms of the above parameters $z_{s}$ and $z_{M}$, i.e. locations of the effective seed and the pumped pulses maxima. For small $\epsilon_{\text {eff }} \ll 1$, the $\pi$-pulse maximum location depends on $\epsilon_{\text {eff }}$ logarithmically, so that changing $\epsilon_{\mathrm{eff}}$ by a factor of two does not affect noticeably the pumped pulse, which remains close to the $\pi$-pulse manifold. This enables reduced description of the pulse evolution in the terms of the pulse projection motion within the $\pi$-pulse manifold. The major goal of this paper is to define the matching $\pi$-pulse parameters and describe their evolution thus extending the quantitative theory of BRA to regimes where the effective seeding power varies substantially during the amplification.

The paper is arranged as follows. First, we show numerically that the pumped pulse indeed can be very well approximated, through the entire advanced nonlinear stage of the amplification, by a pulse growing from a seed of a negligibly short duration and varying effective seeding power $\epsilon_{\text {eff }}$ (Sec. III). Then, we describe analytically (Sec. IV and Sec. V), the motion of the pumped pulse projection within this special manifold of pulses growing from negligibly short seeds. 


\section{BASIC EQUATIONS AND $\pi$-PULSE MANIFOLD}

Resonant Raman backscattering in a plasma can be described by the following equations for the wave envelopes (see, for instance, Refs. [7,3]):

$$
a_{t}+c a_{z}=-\omega_{p} f b, \quad b_{t}-c b_{z}=\omega_{p} a f^{*}, \quad f_{t}=\omega a b^{*} / 2,
$$

where $\omega_{p}$ is the electron plasma frequency, $\omega \gg \omega_{p}$ is the pump laser frequency, $a$ and $b$ are the dimensionless spacetime envelopes of the pump and pumped pulse electric field amplitudes, respectively, normalized so that the pump intensity (i.e., power density) is $I_{a}=\pi c\left(m_{e} c^{2} / e\right)^{2}|a|^{2} / \lambda^{2}=$ $2.736 \times 10^{18}|a|^{2} / \lambda^{2}[\mu \mathrm{m}] \mathrm{W} / \mathrm{cm}^{2}$, with a similar normalization for the seed $b$, while $f$ is an appropriately normalized Langmuir wave envelope; $\lambda$ is the laser wavelength, $t$ is the time, $z$ is the distance in the direction of the pump propagation, subscripts denote the respective derivatives, and $c$ is the vacuum speed of light. The pumped laser frequency is downshifted by $\omega_{p}$ from the pump frequency; the laser wave-numbers are close to $\omega / c$ and the resonant Langmuir wave-number is close to $2 \omega / c$. The slowly varying envelope approximation (1) is applicable as long as the characteristic space and time scales of the wave envelopes substantially exceed $c \omega_{p}^{-1}$ and $\omega_{p}^{-1}$, respectively. We also assume conditions are satisfied allowing to neglect effects of dispersion, diffraction, wave breaking, self-focusing and self-phase-modulation and generation of other harmonics.

For further analysis, it is convenient to rewrite equations (1) in the variables

$$
\zeta=(t+z / c) \sqrt{\omega \omega_{p}} / 2, \quad \tau=-z \sqrt{\omega \omega_{p}} / c, \quad f=\bar{f} \sqrt{\omega / \omega_{p}},
$$

thus getting

$$
a_{\zeta}-a_{\tau}=-\bar{f} b, \quad b_{\tau}=a \bar{f}^{*}, \quad \bar{f}_{\zeta}=a b^{*} .
$$

During the linear stage of the backscattering instability, when the pump depletion is negligible, so that $a \approx a_{0}=$ const, the solution of Eqs. (2) is given by the formulas (Ref. [8,3])

$$
\begin{aligned}
& b(\zeta, \tau)=\frac{\partial}{\partial \zeta} \int d \zeta_{1} G\left(\zeta-\zeta_{1}, \tau\right) b\left(\zeta_{1}, 0\right) \\
& G(\zeta, \tau)=I_{0}(2 \sqrt{\eta}), \quad \eta=a_{0}^{2} \zeta \tau=\xi^{2} / 4
\end{aligned}
$$

where $I_{0}$ is the modified zero-order Bessel function.

During an advanced nonlinear stage of the instability, when the pumped pulse amplification length is much larger than the pulse duration (within which it completely depletes the pump), the term $a_{\tau}$ in (2) can be neglected compared to the term $a_{\zeta}$. Thus simplified, Eqs. (2) conserve the joint density of the pump photons and Langmuir plasmons:

$$
\frac{\partial}{\partial \zeta}\left(|a|^{2}+|\bar{f}|^{2}\right)=0
$$

Real solutions can be then searched in the form

$$
\begin{aligned}
& a(\zeta, \tau)=a_{0} \cos (u / 2), \quad \bar{f}(\zeta, \tau)=a_{0} \sin (u / 2), \\
& b(\zeta, \tau)=u_{\zeta} / 2,
\end{aligned}
$$

which reduces the set to the Sine-Gordon equation

$$
u_{\zeta \tau}=a_{0}^{2} \sin u
$$

This Sine-Gordon equation has a special set of self-similar solutions $u(\zeta, \tau)=U\left(\xi \equiv 2 a_{0} \sqrt{\zeta \tau}\right)$ satisfying the equation

$$
U_{\xi \xi}+U_{\xi} / \xi=\sin U, \quad U(+0)=\epsilon,
$$

and depending on the single parameter $\epsilon$. This is the classical $\pi$-pulse attractor solution (Ref. [5]) that is established at the advanced nonlinear stage of the BRA for seeds of a negligibly short duration.

Note that, for the $\pi$-pulse solution, the relative value of the neglected term $a_{\tau}$ in the energy-containing domain is about $\xi_{M} / a_{0}^{2} \tau^{2}$ (where $\xi_{M}$ is the location of the $\pi$-pulse maximum in self-similar variable $\xi$ ), which indeed quickly decreases as the amplification proceeds.

\section{VARYING EFFECTIVE SEEDING POWER FOR SEEDS OF FINITE-DURATION}

As was noted above, the shadowing of rear layers of seed pulse can affect the actual BRA. For a zero-duration seed $b(z=0, t)=\epsilon \delta(t) / \sqrt{\omega \omega_{p}}=\epsilon \delta(\zeta) / 2$, formally considered within Eqs. (1) or (2), the shadowing effect is absent and the seeding power, constant throughout the amplification length, can be defined as the dimensionless area under the seed envelope $\epsilon=2 \int b(\tau=0, \zeta) d \zeta$. Such zero-duration seed solutions tend asymptotically, at large amplification lengths $-z$, to the $\pi$-pulse manifold.

To define precisely the effective seeding power $\epsilon_{\text {eff }}$ for seeds of finite duration, we formally match the real pumped pulse with the pulse growing from a zero-duration seed and reaching the same maximum pumped amplitude within the same amplification length $-z$ for the same pump amplitude $a_{0}$. The "same amplification length" here is the length traversed by the original seed pulse maximum. Note, that the pumped pulse maximum (trailing the original seed pulse) does not coincide then with the maximum of the pulse growing from a zero-duration seed; the maxima are separated by a distance, which we define as $z_{0}$.

Fig. 1 shows a numerical example of the effective seeding power $\epsilon_{\text {eff }}$ for the Gaussian seed $b(z=0, t)=$ $\epsilon \exp \left[-(t / T)^{2}\right] / T \sqrt{\omega \omega_{p} \pi}$, with $T=2 \pi / \omega_{p}, \epsilon=0.01, \lambda_{p}=$ $2 \pi c / \omega_{p}=12 \mu \mathrm{m}, \lambda=1 \mu \mathrm{m}$ and $a_{0}=0.006$.

Fig. 2 shows the shift $z_{0}$ between the locations of amplitude maximum for the actual pumped pulse and the matching pumped pulse growing from a zero-duration seed, for the same numerical example as above.

To compare shapes of the real and matching pumped pulses (growing from a finite- and zero-duration seeds, respectively) in the above numerical example, we shift the zero-duration seed by $z_{0}$, thus matching the locations of 
maxima for the pumped pulses. Fig. 3 indicates that the pulse envelops indeed coincide very well even for noticeable variations of $\epsilon_{\mathrm{eff}}$.

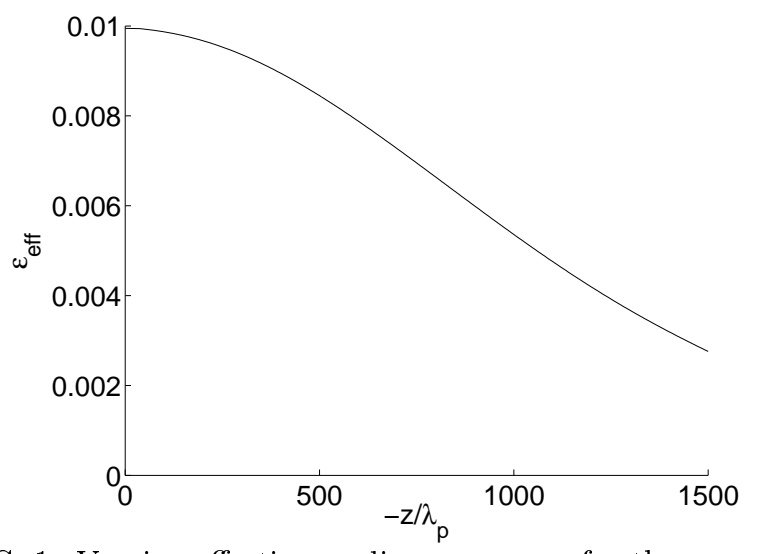

FIG. 1. Varying effective seeding power $\epsilon_{\mathrm{eff}}$ for the Gaussian seed pulse of duration $T=2 \pi / \omega_{p}$ in BRA.

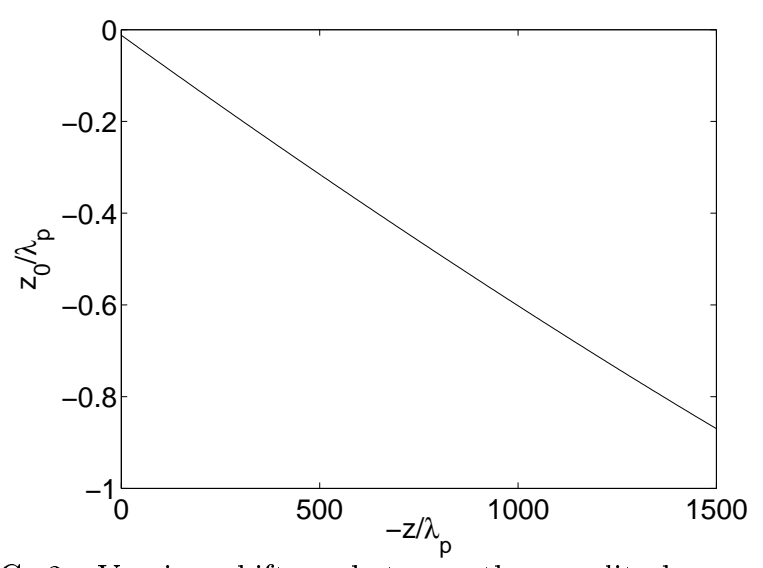

FIG. 2. Varying shift $z_{0}$ between the amplitude maxima of the real and matching pumped pulses (growing from the Gaussian seed pulse of duration $T=2 \pi / \omega_{p}$ and zero-duration seed, respectively).

In Fig. 3, larger rescaled amplitude maxima correspond to larger values of $\epsilon_{\mathrm{eff}}$, i.e., to smaller amplification lengths. The apparent drift of the pumped pulse amplitude maximum to larger $\eta$ in the upper plots (taking place just in the self-similar variable $\eta$ ) attests for the fact that the leading spike of self-similar ( $\pi$-pulse) solution is further from its zero-duration seed (located at $\eta=0$ in these plots) for smaller $\epsilon_{\text {eff }}$. In physical variables $z$ and $t$, the leading spike moving with a superluminous velocity approaches all the time to the zero-duration seed. In lower plots of Fig. 3, placing the Gaussian seed maximum at $\eta=0$, the pumped seed maximum approaches the origin even in self-similar variables. This occurs because, in the process of amplification, the major seeding comes from more and more advanced leading layers of the Gaussian seed.

Note, that using a longer seed would result in an earlier decrease of $\epsilon_{\mathrm{eff}}$. Seeds longer than Raman length $c /\left(a_{0} \sqrt{\omega \omega_{p} / 2}\right)$ would in fact use just the leading the
Raman-length-long part of them right away during the linear stage of the amplification.

Note also, that short Gaussian seeds, like one used above, are favorable for eliminating deleterious superluminous precursors (Ref. [6]).
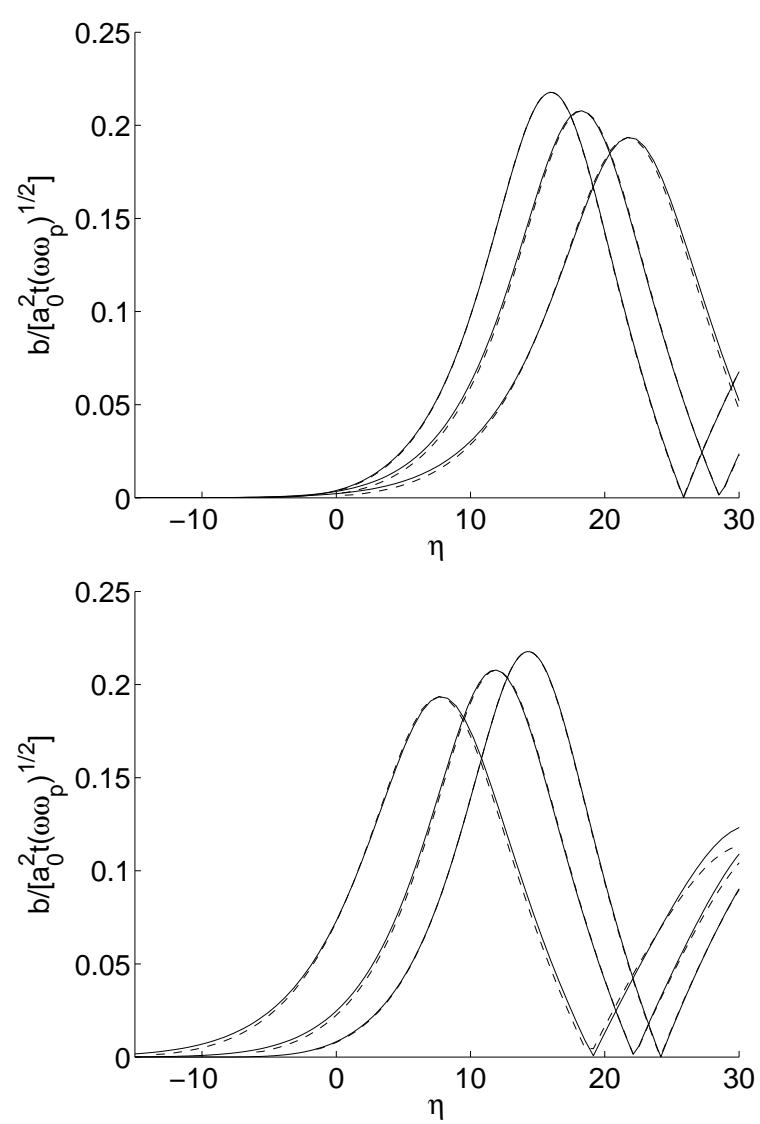

FIG. 3. Rescaled amplitude for the pumped pulse growing from the Gaussian seed pulse of duration $T=2 \pi / \omega_{p}$ (solid lines) and the matching pumped pulse growing from the zero-duration seed (dashed lines) versus $\eta=a_{0}^{2} \zeta \tau=a_{0}^{2} \omega \omega_{p}(t+z / c)(-z / c) / 2$ at the amplification lengths $-z / \lambda_{p}=500 ; 1000 ; 1500$ $\left(\lambda_{p}=2 \pi c / \omega_{p}\right)$. Larger rescaled amplitude maxima correspond to larger values of $\epsilon_{\mathrm{eff}}$, i.e., to smaller amplification lengths. In the upper plots, the matching zero-duration seed is located at $\eta=0$, while in the lower plots, the Gaussian seed maximum is located at $\eta=0$.

\section{SINE-GORDON APPROXIMATION}

As seen from the derivation of Sine-Gordon equation, it can approximate well real solutions of the original equations (1) or (2) at the advanced nonlinear stage of the pulse amplification when the term $a_{\tau}$ in (2) can be neglected, and also during the linear stage of the pulse amplification when the pump variation can be entirely neglected. Thus, there is just a relatively short early nonlinear stage of the pulse amplification when the Sine-Gordon approximation might be not applicable. 
The amplification length around which the complete pump depletion occurs for the first time can be evaluated as follows. During the linear stage, the integrated amplitude $u$ of the pumped pulse can be presented, according to (3) and $(7)$, in the form

$$
u_{l i n}(\zeta, \tau)=2 \int d \zeta_{1} G\left(\zeta-\zeta_{1}, \tau\right) b\left(\zeta_{1}, 0\right),
$$

which gives, for a seed much shorter than the Raman length,

$$
u_{l i n}(\zeta, \tau) \approx \epsilon I_{0}\left(2 a_{0} \sqrt{\zeta \tau}\right) \equiv \epsilon I_{0}(\xi)
$$

For a given $t$, the product $\zeta \tau$ has maximum at $z=-c t / 2$, so that the maximum of integrated amplitude $u$ moves with the speed $-c / 2$ during the linear amplification stage. For a small enough $\epsilon \ll 1$, the pumped pulse makes many exponentiations before reaching the nonlinear stage, which allows one to use the asymptotic formula $I_{0}(\xi) \approx e^{\xi} / \sqrt{2 \pi \xi}$ at $\xi \gg 1$ for an advanced linear stage of the pulse amplification. The same condition allows one to approximate the leading pumped spike in the nonlinear pump depletion regime by the classical $2 \pi$-pulse solution of Sine-Gordon equation. Then, both the linear front and the maximum domains of the leading pumped spike can be described by a single formula (Ref. [1])

$$
u \approx 4 \operatorname{arctg}\left[\epsilon I_{0}(\xi) / 4\right] \approx 4 \operatorname{arctg}\left(u_{l i n} / 4\right)
$$

The condition for the pump complete depletion $u \approx \pi$, reads then as $\epsilon I_{0}(\xi) \approx 4$ thus defining the value $\xi=\xi_{*}$ at which the depletion occurs. This takes place for the first time near the location

$$
-z_{*} \approx c t_{*} / 2 \approx c \xi_{*} / a_{0} \sqrt{2 \omega \omega_{p}}
$$

Numerical comparison of solutions of the original and Sine-Gordon equation indicates that the Sine-Gordon solution does not depart much from the original solution even during the early nonlinear stage of the pulse amplification, as illustrated by Fig. 4.

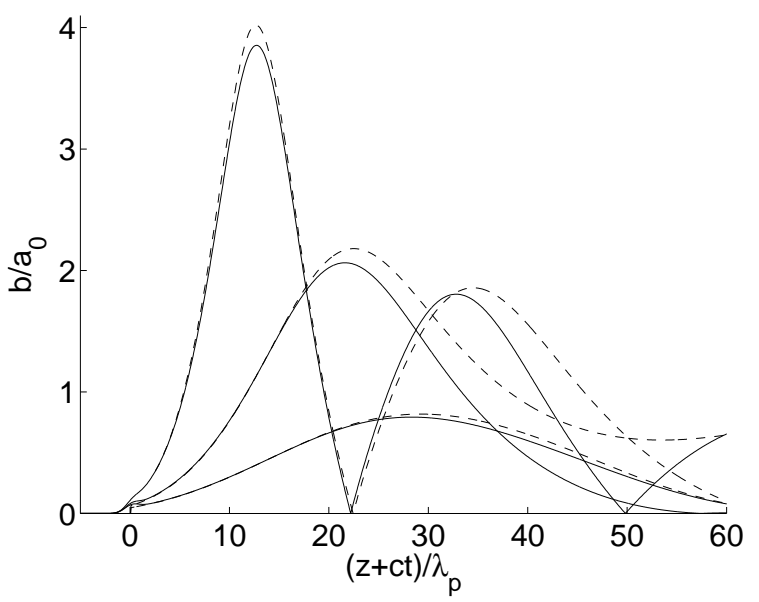

FIG. 4. Sine-Gordon approximation (dashed lines) to the pumped pulse amplitude (solid lines) calculated from the original equations ( 1 ) at $t \omega_{p} / 2 \pi=75 ; 100$; 150 as a function of $(z+c t) / \lambda_{p}$. The seed and pump are the same as above: $a_{0}=0.006, \lambda=1 \mu \mathrm{m}, \lambda_{p}=12 \mu \mathrm{m}$, $b(z=0, t)=\epsilon \exp \left(-t^{2} / T^{2}\right) / T \sqrt{\omega \omega_{p} \pi}, T=2 \pi / \omega_{p}$, $\epsilon=0.01$. The pump complete depletion occurs for the first time at $t \omega_{p} / 2 \pi \approx 100$ which agrees with formula (13).

Thus, the Sine-Gordon approximation appears to be reasonably accurate throughout the entire amplification process. As was noted above, the $\pi$-pulse manifold is the family of self-similar solutions of the Sine-Gordon equation. These solutions correspond to zero-duration seed initial conditions $u(\zeta, \tau=0)=\epsilon \Theta\left(\zeta-\zeta_{s}\right)$, where $\Theta(\zeta)=1$ for $\zeta>0$ and $\Theta(\zeta)=0$ for $\zeta<0$ is the unit step function. It follows that the $\pi$-pulse manifold can also approximate well the entire amplification process. Indeed, the linear and even early nonlinear stages can be approximated by the domain $\xi \lesssim \xi_{*}$ of the $\pi$-pulse, as explained earlier in this section. At advanced nonlinear stage, the $\pi$-pulse is known to be an attractor solution for all $\xi$.

\section{ANALYTICAL APPROXIMATION}

The pump amplitude $a_{0}$ can be excluded from the SineGordon equation (8) by a simple rescaling of independent variable $\bar{\tau}=a_{0}^{2} \tau$. Up to this rescaling, there is a two-dimensional $\pi$-pulse manifold to approximate solutions growing from short yet finite-duration seeds. In this section we derive simple analytical formulas describing dynamics of such solutions projected on the $\pi$-pulse manifold, namely, evolution of the matching zero-duration seed and pumped pulse maximum locations $\zeta_{0}$ and $\zeta_{M}$.

The leading layers of the pumped pulse can be described by the linear theory formula (10). This description can be extended to the nonlinear domain of the leading spike through approximating it, to zeroth order, by the $2 \pi$-pulse, $u \approx 4 \operatorname{arctg}\left(u_{\text {lin }} / 4\right)$. Location of the $2 \pi$-pulse maximum can be determined from the equation $u \approx \pi \Rightarrow u_{\text {lin }} \approx 4$. The respective $\zeta=\zeta_{M}$ satisfies then the equation

$$
\int d \zeta_{1} I_{0}\left(2 \sqrt{\left(\zeta_{M}-\zeta_{1}\right) \bar{\tau}}\right) b\left(\zeta_{1}, 0\right) \approx 2
$$

The integrated function varies rapidly in $\zeta_{1}$, so that the major contribution to the integral comes from a narrow vicinity of this function maximum $\zeta_{1}=\zeta_{01}$. Expanding the logarithm function near the maximum,

$$
S \equiv \ln \left[I_{0}\left(2 \sqrt{\left(\zeta_{M}-\zeta_{1}\right) \bar{\tau}}\right) b\left(\zeta_{1}, 0\right)\right] \approx S_{0}-S_{2}\left(\zeta_{1}-\zeta_{01}\right)^{2}
$$

one can present Eq. (14) in the simplified form

$$
I_{0}\left(2 \sqrt{\left(\zeta_{M}-\zeta_{01}\right) \bar{\tau}}\right) b\left(\zeta_{01}, 0\right) \sqrt{\pi / S_{2}} \approx 2 .
$$

Eq. (16), together with the function $S$ maximum condition, 


$$
\frac{\partial}{\partial \zeta_{01}} \ln \left[I_{0}\left(2 \sqrt{\left(\zeta_{M}-\zeta_{01}\right) \bar{\tau}}\right) b\left(\zeta_{01}, 0\right)\right]=0,
$$

and the definition

$$
S_{2}=-\frac{\partial^{2}}{2 \partial \zeta_{01}^{2}} \ln \left[I_{0}\left(2 \sqrt{\left(\zeta_{M}-\zeta_{01}\right) \bar{\tau}}\right) b\left(\zeta_{01}, 0\right)\right],
$$

define the $\bar{\tau}$-dependent location of the pumped pulse maximum $\zeta_{M}$ together with the auxiliary parameter $\zeta_{01}$. For known $\zeta_{M}$, the effective seed location $\zeta_{0}$ is determined as $\zeta_{0}$ maximizing the product of nonlinear amplification factor $N\left(\zeta_{M}-\zeta_{0}\right)$ and actual seed amplitude $b\left(\zeta_{0}\right)$. It is convenient to present the maximum condition in the form

$$
\frac{\partial}{\partial \zeta_{0}} \ln \left[N\left(\zeta_{M}-\zeta_{0}\right) b\left(\zeta_{0}, 0\right)\right]=0 .
$$

The log-slope of nonlinear amplification factor $N\left(\zeta_{M}-\zeta_{0}\right)$ can be evaluated, in the above $2 \pi$-pulse approximation $u_{\text {lin }} / 4 \approx \operatorname{tg}(u / 4)$ at $u=\pi \Rightarrow u_{\text {lin }} \approx 4$, as

$$
\frac{\partial \ln u}{\partial \zeta} \approx \frac{2}{\pi} \frac{\partial \ln u_{l i n}}{\partial \zeta}
$$

It follows,

$$
\frac{2}{\pi} \frac{\partial}{\partial \zeta_{0}} \ln \left[I_{0}\left(2 \sqrt{\left(\zeta_{M}-\zeta_{0}\right) \bar{\tau}}\right)\right]+\frac{d}{d \zeta_{0}} \ln b\left(\zeta_{0}, 0\right)=0 .
$$

This determines the effective seed location $\zeta_{0}$. A numerical example is presented in Figs. 5 and 6.
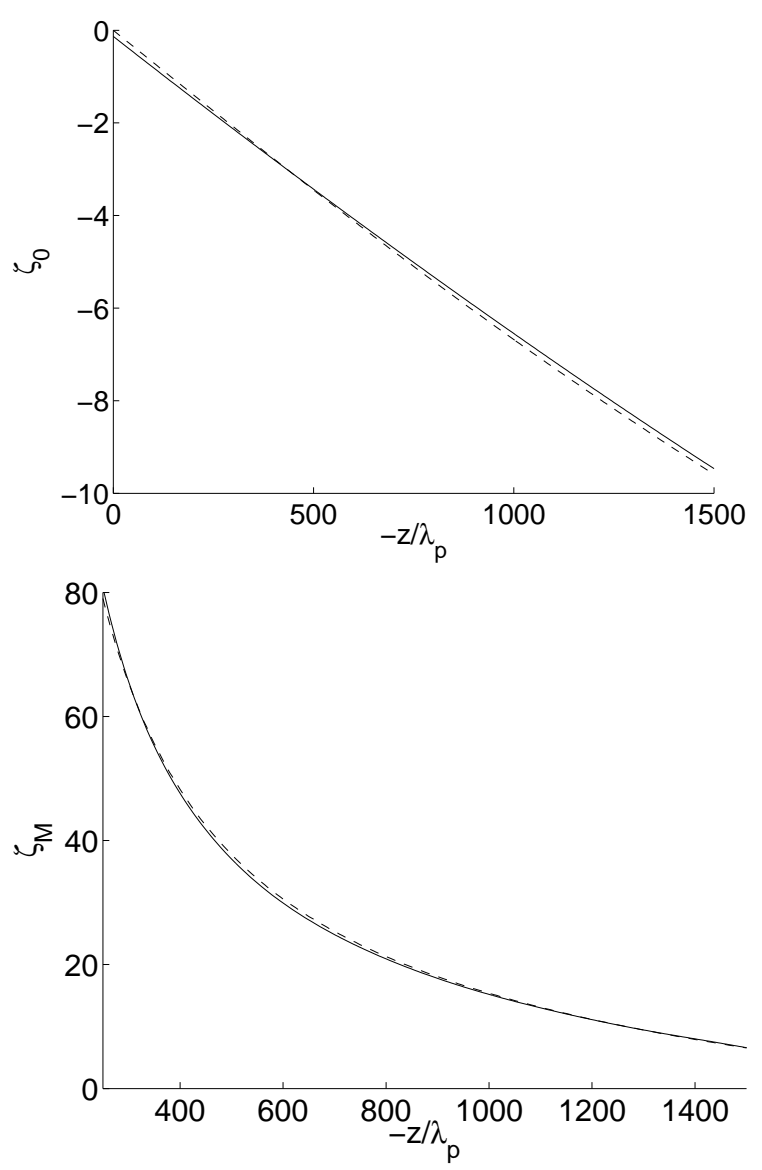

FIG. 5. Locations of the matching zero-duration seed $\zeta_{0}$ (upper figure) and the pumped pulse maximum (in $\zeta$ at fixed $\tau$ or $z$ ) $\zeta_{M}$ (lower figure) as functions of the amplification length $-z$, found numerically as in Sec. III (solid lines) and analytically (dashed lines).

Fig. 5 compares thus calculated parameters $\zeta_{0}$ and $\zeta_{M}$ of the matching $\pi$-pulse and the numerically calculated, as described in the Sec. III, respective parameters of the actual pumped pulse growing from the same as in previous sections Gaussian seed, $b(z=0, t)=\epsilon \exp \left(-t^{2} / T^{2}\right) / T \sqrt{\omega \omega_{p} \pi} \equiv$ $\epsilon \exp \left(-\zeta^{2} / \Delta^{2}\right) / 2 \Delta \sqrt{\pi}$ of the duration $\Delta=T \sqrt{\omega \omega_{p}} / 2=$ $\pi \sqrt{\omega / \omega_{p}}$. Plots for $\zeta_{M}$ are shown just for an advanced nonlinear amplification stage, because, during the linear stage, the pumped pulse amplitude is a monotonically increasing function of $\zeta$ at fixed $\tau$, which is not the case addressed by the analytical theory considered here.

Fig. 6 compares amplitudes of the matching $\pi$-pulse with analytically calculated $\zeta_{0}$ and $\zeta_{M}$ (dashed lines) and of the actual pumped pulse growing from the above Gaussian seed (solid lines). The dotted lines show $\pi$-pulse with $\zeta_{0}$ and $\zeta_{M}$ numerically determined from the actual pumped pulse, as described in Sec. III. The very good coincidence of dotted and dashed lines indicates that their small deviation from solid line at larger $-z$ is not caused by an inaccuracy in calculated $\zeta_{0}$ and $\zeta_{M}$.

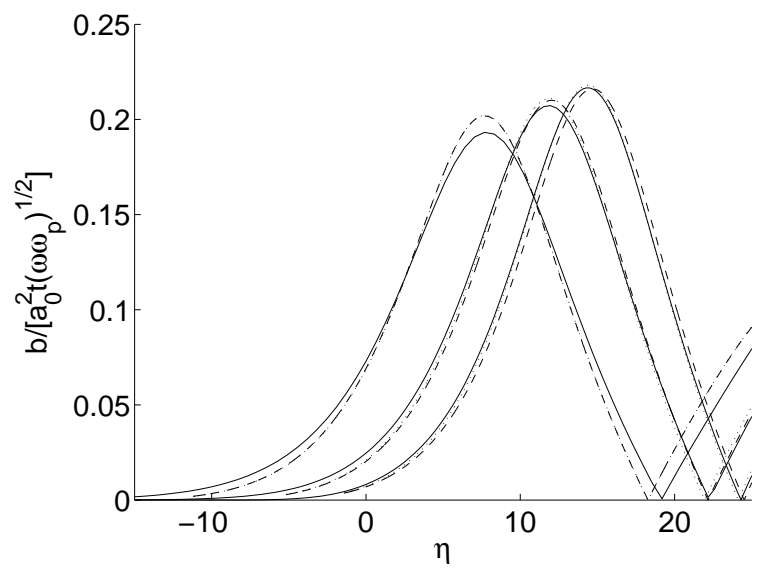

FIG. 6. Normalized amplitude of the actual pumped pulse growing from the Gaussian seed (solid lines), the $\pi$-pulse with analytically calculated $\zeta_{0}$ and $\zeta_{M}$ (dashed lines) and the $\pi$-pulse with $\zeta_{0}$ and $\zeta_{M}$ numerically determined from the actual pumped pulse, as described in Sec. III (dotted lines), for the amplification lengths $-z / \lambda_{p}=500 ; 1000 ; 1500$. Larger maximum amplitudes correspond to larger values of $\epsilon_{\mathrm{eff}}$, i.e., to smaller amplification lengths. The Gaussian seed maximum is located at $\eta=0$.

Note, that using asymptotic formula $I_{0}(\xi) \approx e^{\xi} / \sqrt{2 \pi \xi}$ at $\xi \gg 1$, one could present the equations for calculating parameters $\zeta_{M}$ and $\zeta_{0}$ of the $\pi$-pulse matching the pumped pulse growing from the above Gaussian seed in the following form: 


$$
\begin{aligned}
& \zeta_{01} \approx-\frac{\Delta^{2} \bar{\tau}}{\xi_{M 1}}\left(1-\frac{1}{2 \xi_{M 1}}\right) \\
& \xi_{M 1} \equiv 2 \sqrt{\left(\zeta_{M}-\zeta_{01}\right) \bar{\tau}} \approx \\
& \approx \ln \left[\frac{4}{\epsilon} \sqrt{2 \pi\left(\xi_{M 1}+\frac{2 \Delta^{2} \bar{\tau}^{2}\left(1+\xi_{M 1}^{-1}\right)}{\xi_{M 1}^{2}}\right)}\right]+\frac{\zeta_{01}^{2}}{\Delta^{2}} \\
& \zeta_{0} \approx-\frac{2}{\pi} \frac{\Delta^{2} \bar{\tau}}{\xi_{M}}\left(1-\frac{1}{2 \xi_{M}}\right) \\
& \xi_{M} \equiv 2 \sqrt{\left(\zeta_{M}-\zeta_{0}\right) \bar{\tau}}
\end{aligned}
$$

The lines plotted according to these formulas are nondistinguishable from the dashed lines in Figs. 5.

The approximation of slowly varying $\pi$-pulse parameters is justified as long as the pulse maximum location in selfsimilar variable $\xi_{M}$ does not change much within the amplification length,

$$
\left|\frac{\partial \ln \xi_{M}}{\partial \ln \tau}\right| \ll 1
$$

This condition is satisfied for $\Delta^{2} \bar{\tau}^{2} / \xi_{M}^{3} \ll 1$. Note that at $\bar{\tau} \rightarrow \infty$, the above equations would formally give

$$
\begin{gathered}
\xi_{M 1} \approx(\Delta \bar{\tau})^{2 / 3}, \quad-\zeta_{01} \approx \Delta^{4 / 3} \bar{\tau}^{1 / 3}, \quad-\zeta_{M} \approx 3 \Delta^{4 / 3} \bar{\tau}^{1 / 3} / 4, \\
\xi_{M} \approx 0.72(\Delta \bar{\tau})^{2 / 3}, \quad-\zeta_{0} \approx 0.88 \Delta^{4 / 3} \bar{\tau}^{1 / 3} .
\end{gathered}
$$

Then, $\left|\frac{\partial \ln \xi_{M}}{\partial \ln \tau}\right| \approx 2 / 3$, not satisfying (22), while $\Delta^{2} \bar{\tau}^{2} / \xi_{M}^{3} \approx 2.68$. In our calculations, the parameter $\Delta^{2} \bar{\tau}^{2} / \xi_{M}^{3}$ reaches the value $\approx 0.25$ at $-z=1500 \lambda_{p}$. It still satisfies (22), but approaching to this applicability limit causes a small deviation between theoretical and actual pulses, seen in Fig. 6.

The deviation is even more manifest in the effective seeding power $\epsilon_{\text {eff }}$ that can be calculated from the known $\zeta_{M}-\zeta_{0}$. Within the above asymptotic approximation,

$$
\epsilon_{\mathrm{eff}} \approx 4 \sqrt{2 \pi \xi_{M}} e^{-\xi_{M}} .
$$

Fig. 7 compares this theoretical effective seeding power $\epsilon_{\mathrm{eff}}$ with that numerically calculated as in Sec. III.

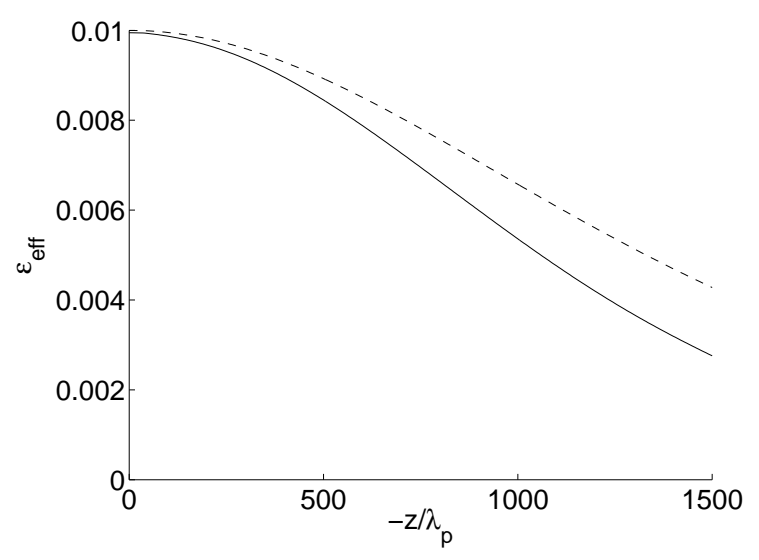

FIG. 7. The effective seeding power $\epsilon_{\text {eff }}$ calculated numerically as in Sec. III (solid line) and analytically (dashed line).

\section{CONCLUSION}

We have described quantitatively the effect of shadowing the rear layers of short laser seeds by the seed leading layers in powerful backward Raman amplifiers (BRA). We have shown that such a shadowing results in the pumped pulse sliding along the manifold of self-similar ( $\pi$-pulse) solutions corresponding to seeds of negligible duration, and derived formulas for parameters of BRA output pulses, taking into account this sliding. Apart from practical importance for precise calculation of BRA output pulses, our results are of interest for general theory of resonant 3 -wave interactions.

\section{ACKNOWLEDGMENT}

This work was supported by DOE DEFY03098UP00210, DOE DEAC02CH03073 and DARPA.

[1] Malkin V. M., Shvets G. and Fisch N. J., Phys. Rev. Lett., 82, 4448 (1999).

[2] G. A. Mourou, C. P. J Barty, M. D. Perry, Phys. Today, 51, 22 (1998). Perry M.D., D. Pennington, B. C. Stuart, et al., Optics Let., 24, 160 (1999).

[3] Malkin V. M., Shvets G. and Fisch N. J., Physics of Plasmas, 7, $2232(2000)$.

[4] G. M. Fraiman, N. A. Yampolsky, V. M. Malkin, and N. J. Fisch, Phys. Plasmas 9, 3617 (2002).

[5] G. L. Lamb, Jr., Phys. Lett. 29A, 507 (1969); Rev. Mod. Phys. 43, 99 (1971); Elements of Solution Theory (Wiley, New York, 1980); V. E. Zakharov, JETP Lett. 32, 589 (1980); S. V. Manakov, JETP Lett., 35, 237 (1982); V. A. Gorbunov, V. B. Ivanov, S. B. Papernyi and V. R. Stratsev, Izv. Akad. Nauk SSSR, Ser. Fez. 48, 1580 (1984) [Bull. Acad. Sci. USSR 48, 120 (1984)]; J. Coste nad C. Montes, Phys. Rev. A 34, 3940 (1986).

[6] Yu. A. Tsidulko, V. M. Malkin, and N. J. Fisch Phys. Rev. Lett., 88, 235004 (2002)

[7] W. L. Kruer, The physics of laser plasma interactions (Addison-Wesley Publ., Redwood City, Calif., 1988), Chapter 7 .

[8] D. L. Bobroff and H. A. Haus, J. Appl. Phys. 38, 390 (1967). 


\section{External Distribution}

Plasma Research Laboratory, Australian National University, Australia

Professor I.R. Jones, Flinders University, Australia

Professor João Canalle, Instituto de Fisica DEQ/IF - UERJ, Brazil

Mr. Gerson O. Ludwig, Instituto Nacional de Pesquisas, Brazil

Dr. P.H. Sakanaka, Instituto Fisica, Brazil

The Librarian, Culham Laboratory, England

Mrs. S.A. Hutchinson, JET Library, England

Professor M.N. Bussac, Ecole Polytechnique, France

Librarian, Max-Planck-Institut für Plasmaphysik, Germany

Jolan Moldvai, Reports Library, Hungarian Academy of Sciences, Central Research Institute for Physics, Hungary

Dr. P. Kaw, Institute for Plasma Research, India

Ms. P.J. Pathak, Librarian, Institute for Plasma Research, India

Ms. Clelia De Palo, Associazione EURATOM-ENEA, Italy

Dr. G. Grosso, Instituto di Fisica del Plasma, Italy

Librarian, Naka Fusion Research Establishment, JAERI, Japan

Library, Laboratory for Complex Energy Processes, Institute for Advanced Study, Kyoto University, Japan

Research Information Center, National Institute for Fusion Science, Japan

Dr. O. Mitarai, Kyushu Tokai University, Japan

Dr. Jiangang Li, Institute of Plasma Physics, Chinese Academy of Sciences, People's Republic of China

Professor Yuping Huo, School of Physical Science and Technology, People's Republic of China

Library, Academia Sinica, Institute of Plasma Physics, People's Republic of China

Librarian, Institute of Physics, Chinese Academy of Sciences, People's Republic of China

Dr. S. Mirnov, TRINITI, Troitsk, Russian Federation, Russia

Dr. V.S. Strelkov, Kurchatov Institute, Russian Federation, Russia

Professor Peter Lukac, Katedra Fyziky Plazmy MFF UK, Mlynska dolina F-2, Komenskeho Univerzita, SK-842 15 Bratislava, Slovakia

Dr. G.S. Lee, Korea Basic Science Institute, South Korea

Institute for Plasma Research, University of Maryland, USA

Librarian, Fusion Energy Division, Oak Ridge National Laboratory, USA

Librarian, Institute of Fusion Studies, University of Texas, USA

Librarian, Magnetic Fusion Program, Lawrence Livermore National Laboratory, USA

Library, General Atomics, USA

Plasma Physics Group, Fusion Energy Research Program, University of California at San Diego, USA

Plasma Physics Library, Columbia University, USA

Alkesh Punjabi, Center for Fusion Research and Training, Hampton University, USA

Dr. W.M. Stacey, Fusion Research Center, Georgia Institute of Technology, USA

Dr. John Willis, U.S. Department of Energy, Office of Fusion Energy Sciences, USA

Mr. Paul H. Wright, Indianapolis, Indiana, USA 
The Princeton Plasma Physics Laboratory is operated by Princeton University under contract with the U.S. Department of Energy.

\author{
Information Services \\ Princeton Plasma Physics Laboratory \\ P.O. Box 451 \\ Princeton, NJ 08543
}

Phone: 609-243-2750

Fax: 609-243-2751

e-mail: pppl_info@pppl.gov

Internet Address: http://www.pppl.gov 